\title{
Powering research reputations: Using real-time reputation building as an incentive to share research discoveries
}

\author{
Courtney Morris* \\ ARTiFACTS, President and Co-founder, 955 Massachusetts Ave \#184, Cambridge, MA 02139, USA
}

\begin{abstract}
The vision for ARTiFACTS sprang from the realization that distributed ledger technology/blockchain technology could be applied directly to scholarly communications. Blockchain fits our current system of citation currency. As Eugene Garfield said decades ago, "The Mertonian description of normal science describes citations as the currency of science. Scientists make payments, in the form of citations, to their preceptors". To work properly, scientists must be able to create, claim ownership, share novel works, and assign proper attribution to prior work at all phases of their research.

However, the existing process of disseminating research and scientific discoveries is skewed towards published articles. Communication, sharing, and the ability to give or receive attribution are delayed far downstream - oftentimes several years - from the actual time when discoveries are made and formally communicated. Distributed ledger technology can deliver transformative value and efficiency in facilitating real-time sharing of the outputs of each step of the research process while providing comprehensive and immutable, break-proof attribution and linking for all relevant research artifacts. Ultimately, this technology will empower the scientific and scholarly communities to affect positive change. This paper briefly discusses some of the early learnings from their recent introduction of the ARTiFACTS system.
\end{abstract}

Keywords: Blockchain, distributed ledger, ARTiFACTS, publishing, data sharing, research attribution

\section{Why ARTiFACTS?}

The research and publishing process is rife with opportunities for improvement. From data access to reproducibility, publishing cycle time to peer review, the challenges have been well-documented and potential solutions fervently debated. Many of these challenges are exacerbated by the constraining nature of today's system.

Multiple years are spent conducting research, which produces many valuable outputs - datasets, observations, study designs and more. This research is winnowed to a single article that is submitted for publication. Then it's a waiting game as the article gets peer-reviewed, (hopefully) published and (sometimes) indexed, which allows the article to be found, referenced and cited. The rest of the research artifacts are never shared - and, therefore, never indexed - so that they are unable to help further the research of other scientists and they cannot participate in building a researcher's reputation.

And for the articles that are published and indexed, there are still several challenges to overcome:

*E-mail: cmorris@artifacts.ai.

0167-5265/18/\$35.00 @ 2018 - IOS Press and the authors.

This article is published online with Open Access and distributed under the terms of the Creative Commons Attribution Non-Commercial License (CC BY-NC 4.0). 
- Inconsistent rules and routines for attribution

- Limited and informal soft metrics

- Citations are subject to breaks and ambiguity

- Disparate systems with dramatically different counts

\section{Giving credit where credit is (over)due}

As Eugene Garfield, the father of bibliometrics, famously said, "Scientists make payments, in the form of citations, to their preceptors". ARTiFACTS seeks to bring that vision to life through a simple, userfriendly platform, purpose built for academic and scientific research.

The ARTiFACTS platform leverages both well-established and innovative technologies, including blockchain, allowing researchers to permanently, securely, and immutably share research artifacts from the earliest stages of research, building their reputation in real-time. By using the ARTiFACTS platform, researchers can:

- Safely provide access to their scientific and academic research outputs

- Irrefutably prove existence of novel work (and, coming soon, prove authorship, not just provenance)

- Easily give and receive real-time attribution for their work, building reputations in real-time

- Comprehensively demonstrate their scholarly contributions

We understand, though, that researchers are reluctant to share work-in-progress for fear of misappropriation. We also know that researchers are also short on time and have no real motivation for sharing outputs. That is why ARTiFACTS makes sharing easy, safe and beneficial.

- Ease of use: ARTiFACTS integrates with leading tools and platforms - within existing researcher workflows and commonly used applications

- Peace of mind: Blockchain enables instant and permanent transactions that provide unquestionable proof of existence and provenance while researchers maintain control of their identity and Intellectual Property

- Reason to act: With an authoritative system for citation management, researchers can provide and receive valid, break-proof attribution and assignment of credit, building their reputation in real time

The result is a single, comprehensive source of truth that is indexed, linked, and searchable, enabling faster, more collaborative research opportunities and more timely contribution to the body of knowledge. Perhaps most importantly, ARTiFACTS provides a more holistic view of the contributions and impact scientists and scholars are making in their fields.

The publishing community benefits as well. Articles that are enhanced with links to supporting content aid in peer review, support reproducibility, and add credibility. This all builds the article's impact, which enhances the journal's reputation.

\section{Just the beginning}

The ARTiFACTS platform continues to grow and evolve as we make improvements and integrate new tools. We've established key partnerships with like-minded organizations passionate about solving the myriad challenges plaguing scholarly research. Recent partnerships seek to: 
- Allow researchers to certify their identity and link it to their ORCID identifier, bringing certainty and simplicity to author identity. Read more about our partnership with the Sovrin Network that was announced in June, 2018

- Integrate blockchain technology into the scholarly publishing workflow with Scienceroot, FractalFlows and Innovative Education Services (ies)

- Collaborate on research to assess the effectiveness of blockchain in healthcare with Blockchain in Healthcare Today and Telehealth and Medicine Today

- Establish the first-ever archive specifically for the population health community Jefferson College of Population Health

Watch for more exciting developments as we continue to build upon and improve the ARTiFACTS platform. To see how the ARTiFACTS platform works, watch our webinar or watch a couple of our short animations.

\section{Behind the scenes}

ARTiFACTS was created by a team of executives with deep experience in primary and secondary academic publishing software, financial services technology, and media. The company's leadership team have all held senior management roles with the world's leading academic research, fintech and educational technology providers, including Thomson Reuters (Web of Science and EndNote), Elsevier (Scopus), ORCID, and SunGard, among others. The company was launched in March 2018 (see press release at: https://www.prnewswire.com/news/ARTiFACTS).

\section{About the Author}

Courtney Morris is a co-founder of ARTiFACTS, the first researcher-specific platform built on blockchain technology. Mr. Morris is a seasoned executive with deep product development, marketing and strategy experience in SAAS and information businesses from education, STM publishing and healthcare. Courtney enjoys skiing and is an instrument rated pilot. Phone: Telephone +1 (617) 902-0622; E-mail: cmorris@artifacts.ai. To learn more about ARTiFACTS go to: artifacts.ai. 